\title{
BMJ Open Prehospital notification of injured patients presenting to a trauma centre in India: a prospective cohort study
}

Biswadev Mitra (1) , ${ }^{1,2,3}$ Vineet Kumar, ${ }^{4}$ Gerard O'Reilly, ${ }^{1,2,3}$ Peter Cameron, ${ }^{1,2,3}$ Amit Gupta, ${ }^{5}$ Amol P Pandit, ${ }^{4}$ Kapil D Soni, ${ }^{5}$ Gaurav Kaushik, ${ }^{5}$ Joseph Mathew, ${ }^{3,6,7}$ Teresa Howard, ${ }^{3,7}$ Madonna Fahey, ${ }^{3}$ Michael Stephenson, ${ }^{2,8,9}$ Satish Dharap, ${ }^{10}$ Pankaj Patel, ${ }^{11}$ Advait Thakor, ${ }^{12}$ Naveen Sharma, ${ }^{13}$ Tony Walker, ${ }^{8}$ Mahesh C Misra, ${ }^{14}$ Russell L Gruen, ${ }^{15}$ Mark C Fitzgerald,,${ }^{3,6,7}$ On behalf of the Australia-India Trauma System Collaboration

To cite: Mitra B, Kumar V, O'Reilly G, et al. Prehospital notification of injured patients presenting to a trauma centre in India: a prospective cohort study. BMJ Open 2020;10:e033236. doi:10.1136/ bmjopen-2019-033236

- Prepublication history and additional material for this paper are available online. To view these files, please visit the journal online (http://dx.doi. org/10.1136/bmjopen-2019033236).

Received 26 July 2019 Revised 13 April 2020 Accepted 06 May 2020
Check for updates

(C) Author(s) (or their employer(s)) 2020. Re-use permitted under CC BY-NC. No commercial re-use. See rights and permissions. Published by BMJ.

For numbered affiliations see end of article.

Correspondence to

Prof Biswadev Mitra;

biswadev.mitra@monash.edu

\section{ABSTRACT}

Objectives To assess the effect of a mobile phone application for prehospital notification on resuscitation and patient outcomes.

Design Longitudinal prospective cohort study with preintervention and postintervention cohorts.

Setting Major trauma centre in India.

Participants Injured patients being transported by ambulance and allocated to red (highest) and yellow (medium) triage categories.

Intervention A prehospital notification application for use by ambulance and emergency clinicians to notify emergency departments (EDs) of an impending arrival of a patient requiring advanced lifesaving care.

Main outcome measures The primary outcome was the proportion of eligible patients arriving at the hospital for which prehospital notification occurred. Secondary outcomes were the availability of a trauma cubicle, presence of a trauma team on patient arrival, time to first chest X-ray, and ED and in-hospital mortality.

Results Data from January 2017 to January 2018 were collected with 208 patients in the preintervention and 263 patients in the postintervention period. The proportion of patients arriving after prehospital notification improved from $0 \%$ to $11 \%(p<0.001)$. After the intervention, more patients were managed with a trauma call-out (relative risk (RR) 1.30; 95\% Cl: 1.10 to 1.52); a trauma bay was ready for more patients (RR $1.47 ; 95 \% \mathrm{Cl}: 1.05$ to 2.05 ) and a trauma team leader present for more patients (RR 1.50; $95 \% \mathrm{Cl}: 1.07$ to 2.10 ). There was no difference in time to the initial chest $X$-ray $(p=0.45)$. There was no association with mortality at hospital discharge (RR 0.94; $95 \% \mathrm{Cl}: 0.72$ to 1.23 ), but the intervention was associated with significantly less risk of patients dying in the $E D$ (RR $0.11 ; 95 \% \mathrm{Cl}: 0.03$ to 0.39 ).

Conclusions The prehospital notification application for severely injured patients had limited uptake but implementation was associated with improved trauma reception and reduction in early deaths. Quality improvement efforts with ongoing data collection using the trauma registry are indicated to drive improvements in trauma outcomes in India.

Trial registration number NCT02877342.

\section{Strengths and limitations of this study}

- Prospective cohort study reporting results of a previously published protocol.

- Data were collected using a newly created trauma registry incorporating prehospital variables.

- The study reports on a novel smartphone-based prehospital notification application.

- The intervention could only be achieved in one of the four planned centres.

- Ongoing improvements in trauma resuscitation and outcomes were potential confounders in the association between prehospital notification and improved processes and outcomes.

\section{INTRODUCTION}

India faces a growing burden from trauma where a person dies from injuries as a result of a road traffic crash every $4 \mathrm{~min}$. The economic burden is substantial. ${ }^{1}$ It is estimated that in urban areas of India, more than $50 \%$ of all trauma deaths could be prevented through improvements in trauma systems. ${ }^{2}$ Inclusive trauma systems focus on all components of patient care (prevention, prehospital care, initial reception and resuscitation, definitive management and rehabilitation). Introduction of trauma systems has been associated with significantly improved outcomes. $^{3} 4$ However, population-based trauma systems in India are generally poorly funded and are struggling to cater for one of the highest population densities in the world. An unknown number of patients are unable to access healthcare, compounded by a lack of universal formal prehospital care services and an organised system of trauma care.

Commencing in 2013, the Australian and Indian Governments invested in a programme to find the best ways of delivering required 
care to injured people. The Australia-India Trauma Systems Collaboration (AITSC) brought together governments, industry, clinicians and researchers to improve information and resources, and to pilot new systems of care. One intervention was the provision for prehospital notification of major trauma patients. The majority of injured patients in India arrive to hospital by private transport. But use of ambulance services is increasing and emergency medicine technicians are qualified to perform initial assessments and provide initial assessment for injured patients. ${ }^{5}$

Prehospital notification of the impending arrival of patients requiring emergency care is seen as an integral component of an advanced prehospital care system. ${ }^{67}$ It is expected that prehospital notification would allow trauma teams more time to prepare, enabling preallocation of tasks and parallel processing of tasks that have been previously demonstrated to improve resuscitation times compared with ad hoc allocation and/or sequential execution of tasks. ${ }^{8}$ However, in the setting of major trauma, limited evidence exists to associate notification with improved patient outcomes. ${ }^{9}$ Prehospital notification was associated with reduced mortality (adjusted OR 0.61, 95\% CI: 0.39 to 0.94; 72073 participants) in Canada. ${ }^{10}$ An improvement in mortality after the introduction of prehospital notification (from $32.4 \%$ in 1987 to $22.7 \%$ 1989) was also reported from the UK. ${ }^{11}$ Improved time to chest X-ray (CXR) has also been associated with prehospital notifications. ${ }^{12}$

The aim of this study was to report on the initial implementation of a prehospital mobile phone applicationbased communication system to enable prehospital care providers to notify the trauma centre of the impending arrival of a seriously injured trauma patient.

\section{METHODS \\ Setting}

The protocol for this study has been previously published. ${ }^{13}$ This study was completed in one of the four planned centres. The Lokmanya Tilak Municipal General Hospital is a Level I trauma centre, which caters to the megapolis of Mumbai and receives about 2500 patients with limb or life-threatening injuries per year.

\section{Patients}

Inclusion and exclusion criteria were consistent with the published protocol; this study included all patients who were transported by a public ambulance and allocated on arrival to hospital to a red (first) or yellow (second) priority triage category after acute trauma, excluding patients allocated to a green (third) category. Patients who were dead at the scene or immediately on arrival without any attempted resuscitation were also excluded. Patients with isolated poisoning, hanging, burns injury only (without other trauma) and single-digit finger or toe amputations (unless of the thumb or great toe) were excluded.

\section{Patient and public involvement}

There was no patient and/or public involvement in the design of the study.

\section{Intervention}

The intervention was the availability of a prehospital notification application named 'Suchana'-an android application for use by ambulance and emergency clinicians in India to notify EDs at selected hospitals of an impending arrival of a patient requiring advanced care. The application is a series of pages with drop-down menus for data entry regarding patient demographics, mechanism of injury and vital signs (figure 1). The application then generates a trauma flag using the previously published algorithm (online supplementary appendix 1 ). ${ }^{13} \mathrm{~A}$ built-in Google maps link determines the estimated time of arrival to the destination hospital. The information is summarised as a prehospital notification to the phone of the receiving trauma hospital (figure 2).

All eligible prehospital clinicians received training on the use of this application which would be accessible from mobile devices such as a tablet, personal computer or smartphone. The training was provided through face-toface sessions over a period of 7 months during the preintervention period, but the application was not available for clinical use. The application used an algorithm based on trauma triage principles developed by the AITSC to derive a trauma priority flag that was automatically transmitted to the hospital. This intervention was accompanied by downstream education of hospital personnel receiving the notification. Education reinforced the importance of patient reception at a trauma bay, involvement of a multidisciplinary trauma team from the outset and initiating assessment and management along Trauma Reception and Resuscitation principles. ${ }^{14}$

\section{Outcomes}

The primary outcome was the proportion of eligible patients arriving at the emergency department (ED), for which prehospital notification occurred. Secondary outcome measures were prespecified to be whether or not there was a trauma call-out, the presence of a trauma bay on arrival, the presence of a trauma team leader, defined in the index hospital to be a third-year surgical resident or senior, time to first CXR, death in the ED and death at hospital discharge.

\section{Analysis}

Continuous data were summarised using mean with SD if normally or near-normally distributed or with medians and IQRs for skewed data. Ordinal data were summarised using medians and IQRs. Nominal, including binary, data were presented as counts with proportions within their categories. Statistical significance was defined as $\mathrm{p}<0.05$. A difference between means was analysed for statistical significance using the Student t-test, whereas the Wilcoxon rank-sum test was used for assessing statistical significance for a difference between medians. The $\chi^{2}$ test 
网

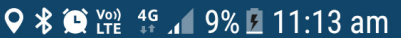

$\equiv \quad$ Edit Patient's Basic Details

○ Single Patient $\bigcirc$ Multiple Patients

Enter Incident ID 及26598

Select Patient's Sex :
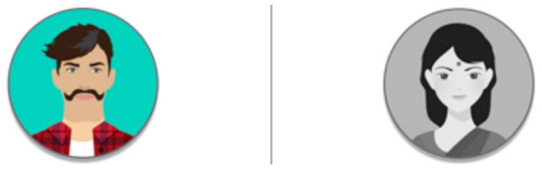

\section{Enter Patient's Age} 30

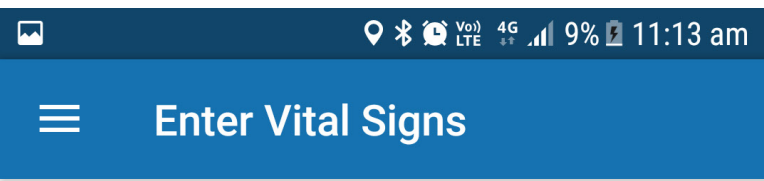

Patient Id 1180 Incident Id 326598

Age

30 Sex

Male

AVPU

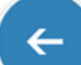

PREV

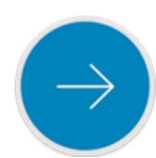

(3)
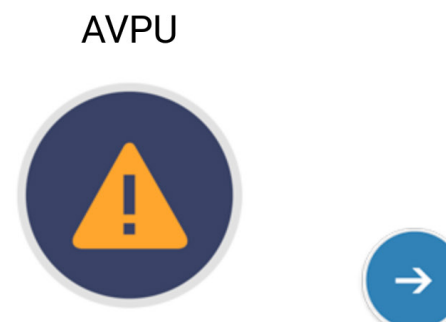

NEXT

\section{Click On Icon For Options}

\begin{tabular}{|c|}
\hline Alert \\
\hline Verbal \\
\hline Responding to Pain \\
\hline Unconscious \\
\hline ETA $: 7$ hours 17 mins \\
\hline
\end{tabular}

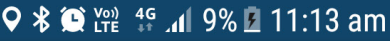

\section{$\equiv \quad$ Enter Vital Signs}

\begin{tabular}{llll} 
Patient Id & 1180 Incident Id & 326598 \\
\hline Age & 30 & Sex & Male
\end{tabular}

\section{MECHANISM OF INJURY}

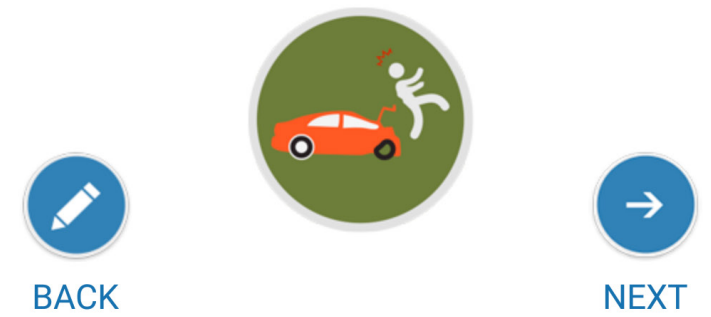

\section{Click On Icon For Options}

\section{Mechanism :}

\section{四

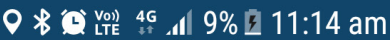 \\ $\equiv \quad$ Review Vital Signs}

Incident Id: 326598

Patient Id: 1180

Age: 30 Sex: Male

\begin{tabular}{ll}
\hline Mechanism & Railway incident \\
\hline Injury Type & Spinal Injury \\
\hline S B P & 123 \\
\hline Heart Rate & 125 \\
\hline Respiratory Rate & 12 \\
\hline AVPU & Verbal \\
\hline Destination & Apollo Hospitals \\
\hline ETA & 7 hours 17 mins
\end{tabular}

\section{Submit To Hospital}

Figure 1 Screenshots of the application for prehospital notification. AVPU: Alert, Verbal, Pain, Unresponsive; SBP: Systolic Blood Pressure; ETA: Estimated time of arrival 


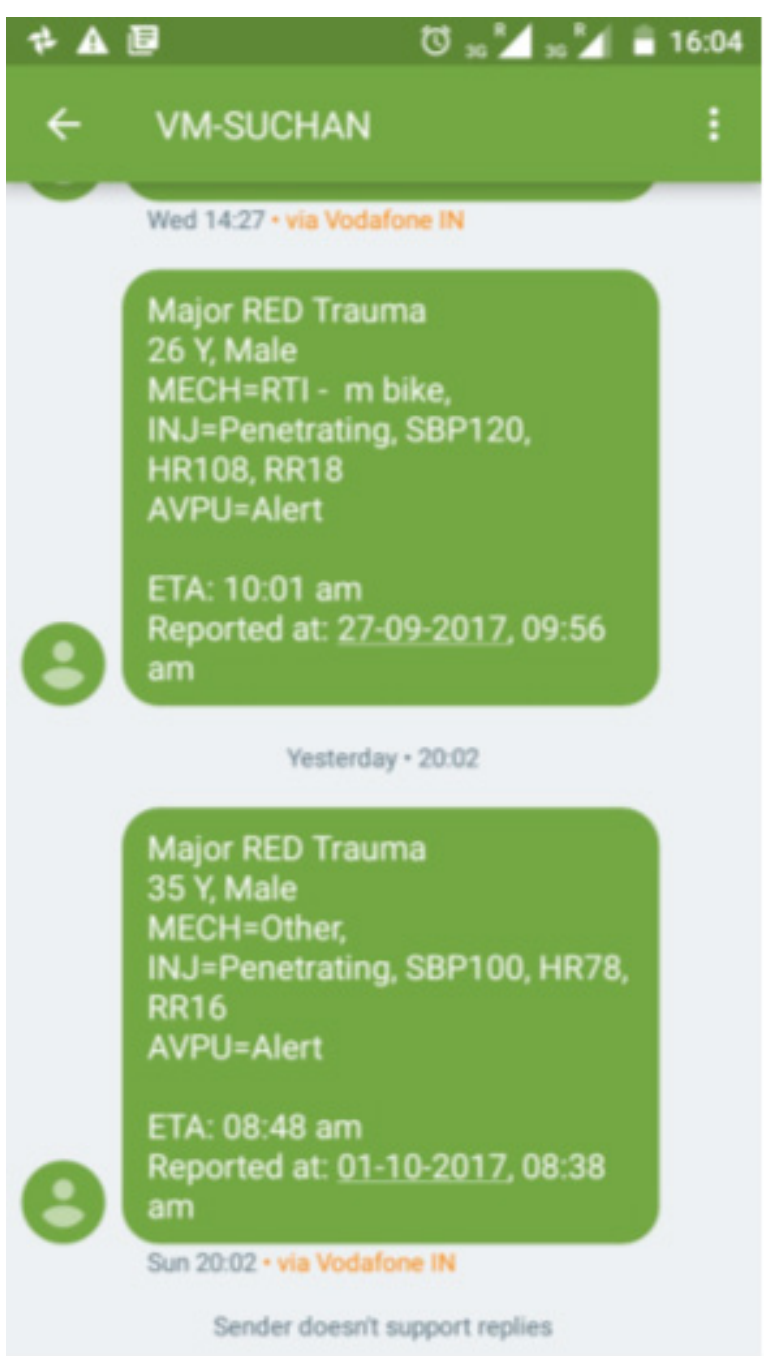

Figure 2 Screenshot of the notification received at the hospital. RR, relative risk. AVPU: Alert, Verbal, Pain, Unresponsive; SBP: Systolic Blood Pressure; ETA: Estimated time of arrival; Inj Mech: Injury Mechanism; RTI: Road traffic incident

or the Fisher exact test (if a cell frequency was $<5$ ) was used for nominal data. The uptake of the intervention over time (with calendar months after the intervention used as units of time) was assessed using an univariable Poisson regression model. Results of this analysis were presenting using an HR (with 95\% CIs) that summarised the association of prehospital notification with introduction of the intervention over time. All analyses were performed using Stata V.15.0 (Statacorp).

The proportion of patients achieving the primary outcome after the intervention was targeted to be 0.70 (achieved by consensus among trauma leaders in Australia and India). This was considered to be a clinically significant level compared with anecdotal reports of current practice of extremely low rates of prehospital notification. The minimum acceptable difference from this hypothesised ideal proportion was considered to be $10 \%$, with a one-sided absolute difference of $>10 \%$ (ie, less than 0.6) to be considered as a failure to achieve the primary outcome. The estimated sample size for a one-sample comparison of proportion with the failure to achieve the primary outcome. The estimated sample size for a one-sample comparison of proportion with the hypothesised value of 0.70 , an alpha of 0.05 and power of 0.90 was 191 after the intervention.

\section{RESULTS}

Data for the preintervention period were gathered from January 2017 to May 2017 and the postintervention period was from June 2017 to January 2018. Within the study period, in this single centre, there were 1613 trauma patients who presented and were triaged to a red or yellow category. In this setting, a high proportion of injured patients arrived by means other than an ambulance with a four-wheeled taxi $(n=557 ; 34.5 \%)$ being the most common mode of transport. Of these, 471 patients were included for analyses with the remaining excluded as they did not present through a mode of transport where the primary intervention of the prehospital notification application was possible (figure 3). There were 208 patients who presented in the preintervention period and 263 patients in the postintervention period.

The mean age of included patients was 32.2 (16.8) years and most patients were of male sex. The most common mechanisms of injury were railway incidents $(n=138$; $29.3 \%)$ and falls $(\mathrm{n}=115 ; 24.4 \%)$. Most included patients were triaged to a red flag $(\mathrm{n}=324 ; 68.8 \%)$. A comparison of patients subgrouped by the primary exposure variable of the availability of the prehospital notification application is presented in table 1 , demonstrating no significant difference among demographics, mechanism of injury and initial vital signs between the two groups.

Outcomes after the intervention of the introduction of a prehospital notification application are listed in table 2. There was a modest, but statistically significant increase in the proportion of patients who arrived at the ED after prehospital notification $(0 \%-11 \% ; \mathrm{p}<0.001)$. The number of events of prehospital notification demonstrated a statistically significant increase over time with a Poisson regression coefficient of 0.77 (95\% CI: 0.60 to $0.95 ; \mathrm{p}<0.001)$. After the intervention, on arrival, more patients were managed with a trauma call-out (relative risk (RR) $1.30 ; 95 \%$ CI: 1.10 to 1.52 ). On arrival to the ED, a trauma bay was ready for more patients (RR 1.47; 95\% CI: 1.05 to 2.05 ) and a trauma team leader present for significantly more patients (RR 1.50; 95\% CI: 1.07 to 2.10) after the intervention. There was no difference in time to initial CXR $(p=0.45)$. On hospital discharge, there was no difference after the intervention in mortality at hospital discharge (RR 0.94; 95\% CI: 0.72 to 1.23 ), but the intervention was associated with significantly less risk of patients dying in the ED (RR 0.11; 95\% CI: 0.03 to $0.39)$.

In the subgroup of 324 patients assigned to a red category, prehospital notification improved from $0 \%$ to $11.6 \%$ $(\mathrm{p}<0.001)$. Improvements were observed in a trauma bay 


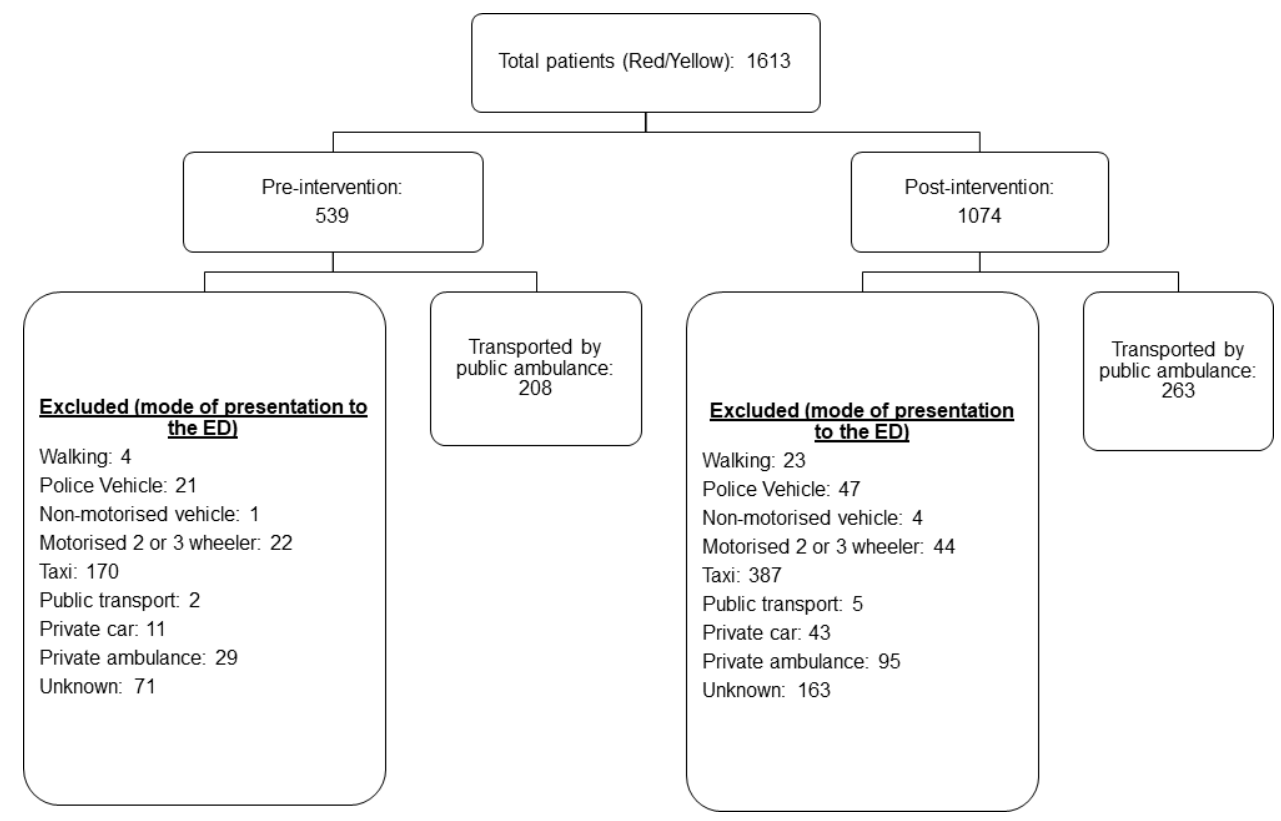

Figure 3 Selection of patients. ED, emergency department.

being ready (RR 1.48; 95\% CI: 1.03 to 2.11 ) and a team leader present on arrival (RR 1.48; 95\% CI: 1.03 to 2.11). There was no association with hospital mortality (RR 0.86 ; 95\% CI: 0.67 to 1.10 ), but there was a significant reduction in the risk of patients' death in the ED (RR 0.10; $95 \%$ CI: 0.03 to 0.35 ).

In the subgroup of 138 patients who were injured through a mechanism involving railways, prehospital notification occurred in $23(27 \%)$ after the intervention. The intervention in this subgroup was associated with more trauma call-outs (RR 2.50; 95\% CI: 1.31 to 4.75 ), team leader presence (RR 2.29; $95 \%$ CI: 1.19 to 4.39 ) and trauma bay being ready on patient arrival (RR 2.29; $95 \%$ CI: 1.19 to 4.39 ). There was no difference in time to GXR, that is, $81 \mathrm{~min}$ (IQR: 58-151) compared with $81.5 \mathrm{~min}(50.5-117.5) \quad(\mathrm{p}=0.44)$. Mortality at hospital discharge remained similar $(43.4 \%$ vs $41.2 \%$; $\mathrm{p}=0.69$ ).

In the subgroup of 29 patients who arrived after notification, $22(76 \%)$ were assigned a red category compared with $302(68.3 \%)$ patients who arrived without notification $(\mathrm{p}=0.40)$. Patients arriving after notification were more frequently injured through a railway incident (76\%) compared with patients arriving without notification $(25.5 \% ; \mathrm{p}<0.001)$. Trauma call-outs were more frequent after notification ( $48.3 \%$ vs $24.4 \%$; $\mathrm{p}=0.005)$, but there was no association with trauma leader presence (37.9\% vs $23.7 \%$; $\mathrm{p}=0.09$ ) or availability of a trauma bay on patient arrival $(37.9 \%$ vs $23.9 \%$; $\mathrm{p}=0.09)$. Time to CXR after notification was 107 min (73-120) compared with 93 min $(54-163)$ without notification $(\mathrm{p}=0.93)$. There were $7(24.1 \%)$ deaths among patients who arrived after notification compared with $140(31.7 \%)$ without notification $(\mathrm{p}=0.40)$.

\section{DISCUSSION}

A prehospital notification system through a smartphone application with downstream education on trauma reception and resuscitation was implemented in a major metropolitan trauma service in India, but with limited uptake of the intervention. After the intervention, there was evidence of improved processes in the initial reception and resuscitation of injured patients through a higher proportion of patients being managed with a trauma call-out, having a trauma bay and trauma team available in the ED on arrival. There was a significant reduction in early deaths in the ED.

However, the study failed to achieve the hypothesised clinically important limit of prehospital notification in at least $70 \%$ of eligible patients. Although a $11 \%$ notification rate was statistically significant (being a change from $0 \%$ ), the study did not achieve the prespecified level of clinical significance. The ambulance system in the city is not run by one organisation. There are multiple charitable and privately operated ambulances that transport patients to hospitals but they were not included in this study. Most of the victims of road or rail traffic injuries were taken to the nearby hospital first and then referred to the trauma centre and may not have been included in this study due to improved haemodynamics. It is possible that a large proportion of patients had short prehospital times and this may have contributed to ambulances not notifying the hospital.

The effective utility of the application required a reliable mobile phone network and anecdotally, there were areas of poor reception within the hospital that may have hampered communication. The public ambulances stationed within a $5 \mathrm{~km}$ radius of the hospital participated in the intervention. However, some patients were transported from outside this radius and hence were not able 
Table 1 Demographic, injury characteristics and presenting vital signs subgrouped by intervention

\begin{tabular}{|c|c|c|c|}
\hline Variable & $\begin{array}{l}\text { Preintervention } \\
(\mathrm{n}=\mathbf{2 0 8})\end{array}$ & $\begin{array}{l}\text { Postintervention } \\
(\mathrm{n}=263)\end{array}$ & $P$ value \\
\hline \multicolumn{4}{|l|}{ Demographics } \\
\hline Age (years) & $33.4(16.8)$ & $31.3(16.7)$ & 0.17 \\
\hline Male sex & $183(88.0 \%)$ & $216(82.1 \%)$ & 0.08 \\
\hline Mechanism of injury & & & 0.79 \\
\hline Motorcar, bus, truck crash & $22(10.6 \%)$ & $24(9.1 \%)$ & \\
\hline Pedestrian/bicycle/tricycle & $5(2.4 \%)$ & $7(2.7 \%)$ & \\
\hline Motorbike crash & $34(16.3 \%)$ & $33(12.5 \%)$ & \\
\hline Autorickshaw & $8(3.8 \%)$ & $9(3.4 \%)$ & \\
\hline Fall from height & $52(25.0 \%)$ & $63(23.9 \%)$ & \\
\hline Penetrating trauma & $9(4.3 \%)$ & $12(4.6 \%)$ & \\
\hline Railway incident & $53(25.5 \%)$ & $85(32.3 \%)$ & \\
\hline Blunt assault & $14(6.7 \%)$ & $22(8.4 \%)$ & \\
\hline Unknown/other & $11(5.3 \%)$ & $8(3.0 \%)$ & \\
\hline \multicolumn{4}{|l|}{ Vital signs (on presentation) } \\
\hline $\begin{array}{l}\text { Systolic blood pressure (mm } \\
\mathrm{Hg})\end{array}$ & $111.2(22.5)$ & $112.1(19.1)$ & 0.65 \\
\hline$<90 \mathrm{~mm} \mathrm{Hg}$ & $13(6.2 \%)$ & $16(6.1 \%)$ & 0.91 \\
\hline Heart rate (bpm) & $96.3(17.2)$ & $97.3(14.8)$ & 0.5 \\
\hline$\geq 120 \mathrm{bpm}$ & $14(6.7 \%)$ & $10(3.8 \%)$ & 0.16 \\
\hline Respiratory rate & $23.8(3.5)$ & $23.8(3.2)$ & 0.99 \\
\hline$<12$ or $>24$ bpm & $37(17.8 \%)$ & $57(21.7 \%)$ & 0.5 \\
\hline GCS on arrival & & & 0.19 \\
\hline $3-8$ & $60(28.8 \%)$ & $69(26.2 \%)$ & \\
\hline $9-12$ & $21(10.1 \%)$ & $42(16.0 \%)$ & \\
\hline $13-15$ & $122(58.6 \%)$ & $151(57.4 \%)$ & \\
\hline Missing & $5(2.4 \%)$ & $1(0.4 \%)$ & \\
\hline Transfer from another hospital & $149(71.6 \%)$ & $203(60.0 \%)$ & 0.17 \\
\hline Arrival trauma flag & & & 0.07 \\
\hline Red & $134(64.4 \%)$ & $190(72.2 \%)$ & \\
\hline Yellow & $74(35.6 \%)$ & $73(27.8 \%)$ & \\
\hline ISS & & & 0.87 \\
\hline $0-12$ & 139 & 168 & \\
\hline $13-24$ & 46 & 66 & \\
\hline $25-44$ & 20 & 24 & \\
\hline$\geq 45$ & 3 & 5 & \\
\hline
\end{tabular}

GCS, Glasgow Coma Scale; ISS, Injury Severity Score.

to notify the hospital. An accurate analysis of factors that contributed to the poor uptake of the intervention was outside the scope of this report and is the aim of future research using a qualitative methodology. We do not believe that technological barriers or lack of resources were responsible as high-quality devices backed by India's extensive mobile networks were used. Potential other reasons could be the need for more training, reluctance of first responders to use the system and improved reception from receiving trauma teams.
Due to the small number of patients who arrived after prehospital notification, statistically significant associations with patient outcomes could not be demonstrated from the single intervention of prehospital notification. Improvements that were observed were most likely due to the combination of prehospital intervention and training in trauma reception and resuscitation and cannot be attributed to a single intervention. The effect of the intervention and accompanying training of prehospital clinicians was likely associated with improved knowledge of 
Table 2 Outcomes

\begin{tabular}{lllc}
\hline Variable & $\begin{array}{l}\text { Preintervention } \\
\text { (n=208) }\end{array}$ & $\begin{array}{l}\text { Postintervention } \\
\text { (n=263) }\end{array}$ & P value \\
\hline $\begin{array}{l}\text { Primary outcome } \\
\text { Prehospital notification }\end{array}$ & 0 & $29(11.0 \%)$ & $<0.001$ \\
$\begin{array}{l}\text { Secondary outcomes } \\
\text { Trauma call-out }\end{array}$ & $40(19.2 \%)$ & $82(31.8 \%)$ & 0.003 \\
Presence of team leader & $40(19.2 \%)$ & $76(28.9 \%)$ & 0.016 \\
Readiness of trauma bay & $41(19.7 \%)$ & $76(28.9 \%)$ & 0.022 \\
\hline Time to first CXR (mins) & $88(56-152)$ & $100(51-153)$ & 0.45 \\
\hline Death at hospital discharge & $67(32.2 \%)$ & $80(30.4 \%)$ & 0.68 \\
\hline Death in the ED & $20(9.6 \%)$ & $3(1.1 \%)$ & $<0.001$ \\
\hline
\end{tabular}

*Three hundred and eighteen patients had a CXR in the ED.

CXR, chest X-ray; ED, emergency department.

injury severity. Contributory factors toward the significant reduction in early deaths are most likely multifactorial and complemented by a structured trauma reception and resuscitation process. It may be an indication that resuscitation attempts were better sustained in the short term resulting in the better disposition of the patient to the operating room or intensive care unit.

Previous studies evaluating trauma outcomes in India have been limited, with the absence of prehospital data and the inability to evaluate the influence of prehospital factors on trauma outcomes. ${ }^{15}$ There was a significant trend towards improved uptake of the intervention, although within the limitations of low numbers. Our results demonstrated a $77 \%$ improvement in the number of prehospital notifications over each month and we are $95 \%$ confident that this value lies between $60 \%$ and $95 \%$. This intervention, however, did not improve the documentation of prehospital variables for the assessment of trauma outcomes. However, in-hospital data from the registry provided robust data, collected prospectively, during the initial hospital reception and resuscitation phase. This study confirms the importance of improving processes across the whole journey of a major trauma patient. Although prehospital notification and initial improvements in trauma reception may improve processes and reduce errors, they have not been previously associated with in-hospital outcomes. ${ }^{14}$

The unique epidemiology of trauma in India and geographic variations have been previously highlighted, with a disproportionate burden of major trauma from railway incidents. ${ }^{16} 17$ The high mortality from this mechanism (in excess of 40\%) highlighted an area for urgent intervention. It was encouraging to observe improvements in the prehospital notification for railway incidents and a team-based reception of patients, but it is obvious that more system-wide prevention and management strategies are required to improve outcomes of this important subgroup.

The poor initial uptake of this intervention by prehospital public ambulances should not be a deterrent to continuing efforts to improve prehospital communication in the Indian system. An extremely high mortality rate of over $30 \%$ among patients who were transported to the ED alive confirms the urgent need for improved trauma systems in India. Prehospital services are rapidly expanding across India with over 20 states having some form of Government or Private-Public Partnership prehospital/emergency service. India is rapidly moving toward a Western model of prehospital service with the aim that all major trauma patients will arrive by an ambulance staffed by personnel trained in basic or advanced life support. In addition, a high proportion of patients arrived by other means, necessitating prehospital communication tools that can be applied to a wide range of services. This finding presents opportunities for basic training in recognition of severe injury prehospital notification for taxi drivers and police personnel.

This study was limited in being confined to a single centre and completed in only one of the four planned sites. However, the required sample size was achieved in the single site. Due to the limited uptake of the intervention, complete prehospital data were not available in the registry, limiting analysis of the association between prehospital clinical variables and outcomes. The reasons for the low prehospital notification rates could not be identified and require a qualitative assessment of prehospital providers' opinions towards the intervention. This should be considered as the focus of future research and essential to elucidate the reasons for initial low uptake. Potential strategies to increase prehospital notification include ongoing training, linking of prehospital notification to key performance indicators and ongoing research demonstrating improved outcomes through improved communication between prehospital and in-hospital staff. ${ }^{18}$ Improvement measures in communication skills through education of prehospital personnel in India have been previously demonstrated and could be expanded to include identification and notification of severely injured patients. ${ }^{19} 20$ Secondary outcome variables of the presence of trauma team leader, trauma bay availability and 
time to CXR were selected as they were assumed to have a direct effect from the prehospital notification. Other outcome measures of effective trauma receptions such as time to theatre or time to haemostasis are important patient-centric outcomes but have multiple potential confounders that were not assessed in this trial. These could be the focus of future studies.

\section{CONCLUSIONS}

A prehospital notification application for severely injured trauma patients had limited uptake. However, the introduction along with training in trauma reception and resuscitation was associated with improved trauma reception and reduction in early deaths after trauma. Ongoing quality improvement efforts with ongoing data collection using the trauma registry are indicated to drive improvements in trauma outcomes in India.

\section{Author affiliations}

${ }^{1}$ Emergency \& Trauma Centre, The Alfred Hospital, Melbourne, VIC, Australia

${ }^{2}$ School of Pulic Health and Preventive Medicine, Monash University, Melbourne, VIC, Australia

${ }^{3}$ National Trauma Research Institute, The Alfred Hospital, Melbourne, VIC, Australia

${ }^{4}$ Department of Surgery, Lokmanya Tilak Municipal Medical College and General Hospital, Mumbai, Maharashtra, India

${ }^{5}$ JPN Apex Trauma Centre, All India Institute of Medical Sciences, New Delhi, Delhi, India

${ }^{6}$ Trauma Service, The Alfred Hospital, Melbourne, VIC, Australia

${ }^{7}$ Central Clinical School, Monash University, Melbourne, VIC, Australia

${ }^{8}$ Ambulance Victoria, Doncaster, VIC, Australia

${ }^{9}$ Department of Community Emergency Health and Paramedic Practice, Monash University, Melbourne, VIC, Australia

${ }^{10}$ Department of Surgery, Topiwala National Medical College \& B.Y.L. Nair Ch.

Hospital, Mumbai, Maharashtra, India

${ }^{11}$ Department of Orthopaedic Surgery, Smt NHL Municipal Medical College, Ahmedabad, Gujarat, India

${ }^{12}$ Department of Emergency Medicine, Smt NHL Municipal Medical College, Ahmedabad, Gujarat, India

${ }^{13}$ Department of General Surgery, All India Institute of Meical Sciences Jodhpur, Jodhpur, Rajasthan, India

${ }^{14}$ Surgical Disciplines, Mahatma Gandhi University of Medical Sciences and Technology, Jaipur, Rajasthan, India

${ }^{15}$ College of Health \& Medicine, Australian National University, Canberra, ACT, Australia

Twitter Biswadev Mitra @Biswadev_M, Peter Cameron @prof_cameron, Naveen Sharma @indiansurgeon and Mark C Fitzgerald @markcfitz

Acknowledgements The authors would like to thank staff at the recruiting hospital and data collectors for their assistance in the completion of the project. GO'R is currently an NHMRC Professional Research Fellow at the National Trauma Research Institute, Melbourne.

Contributors The project was planned by RG, MCF, MCM, AG and AT. Study design was undertaken, in consultation with all authors, by BM, VK, APP, PC, TH, MF and JM. Site-specific processes were arranged and processes unique to each site added to the manuscript by KDS, GK, NS, SD and PP. Biostatistician GO'R reviewed the proposal and provided input to research design and analysis. MS and TW provided expert opinion on prehospital systems. All authors reviewed and edited the final manuscript.

Funding The AITSC was funded by the Indian Government (Department of Science and Technology) and the Australian Government (Department of Industry, Innovation and Science), through the Australia-India Strategic Research Fund (AISRF), Grand Challenge Round 2, AISRF-GA12, Grant Number GCF0200130.

Competing interests None declared.
Patient consent for publication Not required.

Ethics approval The study was reviewed and ethical approval obtained from the All India Institute of Medical Sciences (AllMS), New Delhi, India, and site-specific approval has been acquired from the relevant trauma services. The project was reviewed by the Monash University Human Research Ethics Committee and approval granted; (Project number: CF16/1814-2016000929). The requirement to seek informed consent from participants was waived by the ethics committees.

Provenance and peer review Not commissioned; externally peer reviewed.

Data availability statement Data are available upon reasonable request. Data may be made available on request subject to approval by relevant ethics committees.

Open access This is an open access article distributed in accordance with the Creative Commons Attribution Non Commercial (CC BY-NC 4.0) license, which permits others to distribute, remix, adapt, build upon this work non-commercially, and license their derivative works on different terms, provided the original work is properly cited, appropriate credit is given, any changes made indicated, and the use is non-commercial. See: http://creativecommons.org/licenses/by-nc/4.0/.

ORCID iD

Biswadev Mitra http://orcid.org/0000-0002-0508-2450

\section{REFERENCES}

1 Goli S. Road traffic accidents and injuries in India.

2 Roy N, Kizhakke Veetil D, Khajanchi MU, et al. Learning from 2523 trauma deaths in India- opportunities to prevent in-hospital deaths. BMC Health Serv Res 2017;17:142.

3 Gabbe BJ, Simpson PM, Sutherland AM, et al. Improved functional outcomes for major trauma patients in a regionalized, inclusive trauma system. Ann Surg 2012;255:1009-15.

4 Lockey DJ. Improved trauma outcomes after the introduction of a trauma system in England. EClinicalMedicine 2018;2-3:3-4.

5 Newberry JA, Bills CB, Matheson L, et al. A profile of traumatic injury in the prehospital setting in India: a prospective observational study across seven states. Injury 2020;51:286-93.

6 Cameron PA, Gabbe BJ, Smith K, et al. Triaging the right patient to the right place in the shortest time. Br J Anaesth 2014;113:226-33.

7 Johnson D, Wilson M. Improving prehospital information for trauma patients. Australian Emergency Nursing Journal 1998;1:12-13.

8 Driscoll PA, Vincent CA. Organizing an efficient trauma team. Injury 1992;23:107-10.

9 Synnot A, Karlsson A, Brichko L, et al. Prehospital notification for major trauma patients requiring emergency Hospital transport: a systematic review. J Evid Based Med 2017;10:212-21.

10 Liberman M, Mulder DS, Jurkovich GJ, et al. The association between trauma system and trauma center components and outcome in a mature regionalized trauma system. Surgery 2005; 137:647-58.

11 Maimaris C, Brooks SC. Monitoring progress in major trauma care using TRISS. Emergency Medicine Journal 1990;7:169-71.

12 Lillebo B, Seim A, Vinjevoll O-P, et al. What is optimal timing for trauma team alerts? A retrospective observational study of alert timing effects on the initial management of trauma patients. $J$ Multidiscip Healthc 2012;5:207-13.

13 Mitra B, Mathew J, Gupta A, et al. Protocol for a prospective observational study to improve prehospital notification of injured patients presenting to trauma centres in India. BMJ Open 2017;7:e014073.

14 Fitzgerald Met al. Trauma resuscitation errors and computer-assisted decision support. Arch Surg 2011;146:218-25.

15 Murlidhar V, Roy N. Measuring trauma outcomes in India: an analysis based on TRISS methodology in a Mumbai university hospital. Injury 2004;35:386-90.

16 Laytin AD, Kumar V, Sarang B, et al. Patterns of injury mechanism at a tertiary trauma center in Mumbai, India: opportunities for injury prevention. J Transport Safety Sec 2017;9:103-14.

17 Mohanty MK, Panigrahi MK, Mohanty S, et al. Death due to traumatic Railway injury. Med Sci Law 2007;47:156-60.

18 Gruen RL, Gabbe BJ, Stelfox HT, et al. Indicators of the quality of trauma care and the performance of trauma systems. Br J Surg 2012;99 Suppl 1:97-104.

19 Aekka A, Abraham R, Hollis M, et al. Prehospital trauma care education for first responders in India. J Surg Res 2015;197:331-8.

20 Vyas D, Hollis M, Abraham R, et al. Prehospital care training in a rapidly developing economy: a multi-institutional study. J Surg Res 2016;203:22-7. 